\title{
Robotic Surgery-A New Paradigm in Head \& Neck Surgery
}

\section{Rohan R Walvekar*, Evan A. Longfield and Elliot J. Scott}

Department of Otolaryngology Head \& Neck Surgery, LSU Health Sciences Center, New Orleans, LA 70112, USA

The field of otolaryngology has always looked towards technology for advances in medical therapeutics. The anatomical contours and complexities of this region, as well as the delicate structures and proximity of these to each other, pose diagnostic, therapeutic, and surgical challenges. The use of endoscopes, for example, revolutionized the management of sinus disease. The introduction of the da Vinci surgical system and the application of robotics within head and neck surgery has been one of the most exciting developments within the field of head and neck surgery in the recent past.

One of the most significant criticisms to surgical management of head and neck tumors, especially tumors of the tonsil and base of the tongue, has been the need for large access procedures that require lip splitting or mandibulotomy to visualize tumors. In addition to the inherent morbidity of the procedures, there is also a risk of seeding malignancy by delivering the pharyngeal contents into the neck.

Furthermore, these traditional approaches have left patients with complicated or disfiguring defects that require complex reconstructions with local, regional, or free tissue transfer. Although these procedures have proved effective to varying degrees in achieving local and regional disease control, the functional impact on speech and swallowing dysfunction is significant [1-3]. Optics and instrumentation with multiple degrees of rotation, of the da Vinci robotic system permits surgical intervention for oropharyngeal lesions, without the need for large access procedures, while maintaining surgical dexterity and accuracy. In addition to this, the three-dimensional camera can be positioned close to the tumor, thus providing an excellent view of the surgical bed [3].

The application of robotically assisted surgery in the head and neck began in 2003 when McLeod et al. [4], performed the first transoral experiments with the da Vinci system in both porcine and cadaveric models using a Lindholm retractor. They found that the robot offered several advantages including: lower morbidity, greater freedom of movement, and an enhanced three-dimensional view. The concept of Transoral Robotic Surgery (TORS) was further established at the University of Pennsylvania, when Hockstein et al. demonstrated wide access to the laryngopharynx using oropharyngeal retractors in an airway mannequin and cadaver [5]. Weinstein et al. later performed a supraglottic laryngectomy in a canine model. He demonstrated increased exposure using an oropharyngeal retractor, with adjustable visualization of the larynx to facilitate the resection [6,7].

The first case series of patients undergoing TORS for oropharyngeal cancer (OPC) was also reported from the University of Pennsylvania [8]. Three patients with early stage, base of tongue squamous cell carcinomas underwent complete en bloc resection of their tumors with negative margins. No immediate complications were noted, and patients were able to return to a full diet within six weeks of surgery.

Long-term oncologic outcomes of TORS are not possible at this point because of the relative infancy of the procedure. However, several institutions have published short-term data from small case series [912]. These early evaluations of the oncological and functional outcomes of TORS illustrate a minimally invasive technique that permits resection of the tumor en bloc while preserving patients' swallowing ability. In
December 2009, the promising results of the data from these multiple institutions led the FDA to approve TORS for use in selected benign and malignant tumors of the head and neck.

Recently a three-center study (conducted at the University of Pennsylvania, the Mayo Clinic, and the University of Alabama Birmingham) evaluated and reported the safety and efficacy of TORS [13]. This 177 patient study concluded that TORS demonstrated a very high safety and efficacy profile. They reported a positive margin rate of $4.3 \%$. Only $16 \%$ experienced serious adverse events that required hospitalization or intervention postoperatively. Tracheostomy was performed in $12.4 \%$ of all patients, but only $2.3 \%$ had a tracheostomy at their last follow-up. For all patients undergoing TORS without previous therapy, the percutaneous endoscopic gastrostomy dependency rate was $5.0 \%$. Lastly, the average hospital stay was 4.2 days. This study's encouraging results drew the attention of the scientific community, which led to the re-emergence of surgical therapy and individualizing treatment protocols for patients newly diagnosed with cancers of the oropharynx.

Although the application of robotics in the head and neck was conceptualized for easy access to difficult-to-reach areas in the oropharynx, its success as a surgical device created the opportunity for various other head and neck applications. TORS heralded the existence of robotics as part of a growing field of endoscopic head and neck surgery (eHNS). This continuum today includes various innovative applications of the robot for management of benign tumors of the oropharynx and parapharyngeal space [14]. There is also experimental data looking into the feasibility of robotic surgery for management of skull base tumors $[15,16]$.

One of the most exciting new advances is the utilization of the robot for distant access thyroid and parathyroid surgery. This provides safe access to the neck compartment in select cases and makes possible safe dissection of critical and delicate neck contents from a distant site, in this case the axillary crease. Furthermore, the recent emergence of transaxillary thyroidectomy not only provides an exciting new approach to thyroid resection, but may also prove to be a stepping stone to minimally invasive cervical lymph node dissection [17].

In otolaryngology, the introduction of robotic surgery has been received with enthusiasm as well as speculation. It is natural to question

*Corresponding author: Rohan R. Walvekar, Associate Professor, Department of Otolaryngology Head \& Neck Surgery, Louisiana State University Health Sciences Center, 533 Bolivar Street, Suite 566, New Orleans, LA 70112, USA, Email: rwalve@lsuhsc.edu

Received October 24, 2012; Accepted October 27, 2012; Published October 30 , 2012

Citation: Walvekar RR, Longfield EA, Scott EJ (2012) Robotic Surgery-A New Paradigm in Head \& Neck Surgery. Adv Robot Autom S7:e001. doi:10.4172/21689695.S7-e001

Copyright: (c) 2012 Walvekar RR, et al. This is an open-access article distributed under the terms of the Creative Commons Attribution License, which permits unrestricted use, distribution, and reproduction in any medium, provided the original author and source are credited. 
Citation: Walvekar RR, Longfield EA, Scott EJ (2012) Robotic Surgery-A New Paradigm in Head \& Neck Surgery. Adv Robot Autom S7:e001. doi:10.4172/2168-9695.S7-e001

the utility of novel technology and how it applies to different specialties. Our experience implementing robotic surgery into our practice has been rewarding. We have found TORS not only to be safe and effective for the treatment of oropharyngeal tumors, but also to have many advantages operating within the oral cavity $[18,19]$. These observations led us to innovate by using the robot for novel indications in the anterior and lateral floor of the mouth. We have demonstrated that floor of mouth tumors and delicate structures, such as the lingual nerve and salivary duct, are amenable to robotic surgical dissection. We have demonstrated that ranulas, both floor of mouth and plunging ranulas as well as large submandibular stones (megaliths), can be safely managed in a minimally invasive fashion using the robot. This approach has the combined advantage of preserving the gland and avoiding an external scar. We have also found robotically-assisted trans-axillary approaches to the neck to be safe and effective treatment for patients with isolated, unilateral thyroid and parathyroid neoplasms with low malignant potential.

We believe we are only just beginning to uncover the possibilities of robot-assisted surgery in the head and neck. Advances in instrumentation, integration of imaging, and further miniaturization of surgical tools will allow us greater access to tumors that could not previously be treated surgically. However, as we embrace new technology and incorporate novel surgical techniques, we must remember our responsibility to constantly audit our actions and ensure that we provide equivalent, if not better care to our patients that is safe and cost-effective.

\section{References}

1. Garg A, Dwivedi RC, Sayed S, Katna R, Komorowski A, et al. (2010) Robotic surgery in head and neck cancer: a review. Oral Oncol 46: 571-576.

2. Parmar A, Grant DG, Loizou $P$ (2010) Robotic surgery in ear nose and throat. Eur Arch Otorhinolaryngol $267: 625-633$.

3. Weinstein GS, O'Malley BW, Desai SC, Quon H (2009) Transoral robotic surgery: does the ends justify the means? Curr Opin Otolaryngol Head Neck Surg 17: 126-131.

4. McLeod IK, Mair EA, Melder PC (2005) Potential applications of the da Vinci minimally invasive surgical robotic system in otolaryngology. Ear Nose Throat J 84: 483-487.

5. Hockstein NG, Nolan JP, O'malley BW, Woo YJ (2005) Robotic microlaryngeal surgery: a technical feasibility study using the daVinci surgical robot and an airway mannequin. Laryngoscope 115:780-785.

6. Weinstein GS, O'malley BW, Hockstein NG (2005) Transoral robotic surgery: supraglottic laryngectomy in a canine model. Laryngoscope 115: 1315-1319.

7. Weinstein GS, O'Malley BW, Snyder W, Hockstein NG (2007) Transoral robotic surgery: supraglottic partial laryngectomy. Ann Otol Rhinol Laryngol 116: 19-23.

8. O'Malley BW, Weinstein GS, Snyder W, Hockstein NG (2006) Transoral robotic surgery (TORS) for base of tongue neoplasms. Laryngoscope 116: 1465-1472.

9. Weinstein GS, O'Malley BW, Snyder W, Sherman E, Quon H (2007) Transoral robotic surgery: radical tonsillectomy. Arch Otolaryngol Head Neck Surg 133 1220-1226.

10. Boudreaux BA, Rosenthal EL, Magnuson JS, et al. (2009) Robot-assisted surgery for upper aerodigestive tract neoplasms. Arch Otolaryngol Head Neck Surg 135: 397-401.

11. Moore EJ, Olsen KD, Kasperbauer JL (2009) Transoral robotic surgery for oropharyngeal squamous cell carcinoma: a prospective study of feasibility and functional outcomes. Laryngoscope 119:2156-2164.

12. Genden EM, Desai S, Sung CK (2009) Transoral Robotic Surgery For The Management Of Head And Neck Cancer: A Preliminary Experience. Head \& neck 31: 283-289.

13. Weinstein GS, O'Malley BW, Magnuson JS, Carroll WR, Olsen KD, et al. (2012) Transoral robotic surgery: A multicenter study to assess feasibility, safety, and surgical margins. Laryngoscope 122: 1701-1707.

14. O'Malley BW, Quon H, Leonhardt FD, Chalian AA, Weinstein GS (2010) Transoral robotic surgery for parapharyngeal space tumors. ORL J Otorhinolaryngol Relat Spec 72:332-326.

15. O'Malley BW jr, Weinstein GS (2007) Robotic anterior and midline skull base surgery: preclinical investigations. Int J Radiat Oncol Biol Phys 69: S125-S128.

16. Lee JYK, O’Malley BW, Newman JG, et al. (2010) Transoral robotic surgery of the skull base: a cadaver and feasibility study. ORL; ORL J Otorhinolaryngo Relat Spec 72: 181-187.

17. Holsinger FC, Sweeney AD, Jantharapattana K, Salem A, Weber RS, et al. (2010) The emergence of endoscopic head and neck surgery. Curr Oncol Rep 12: $216-222$.

18. Walvekar RR, Peters G, Hardy E, Alsfeld, L, Stromeyer FW, et al. (2011) Robotic-assisted transoral removal of a bilateral floor of mouth ranulas. World J Surg Oncol 9: 78

19. Walvekar RR, Tyler PD, Tammareddi N, Peters G (2011) Robotic-assisted transoral removal of a submandibular megalith. Laryngoscope. 121: 534-537.
This article was originally published in a special issue, Robotics and Advances in Medicine handled by Editor(s). Dr. Rohan Ramchandra Walvekar, Mary Bird Perkins Cancer Center, USA 